\title{
Real-time Real-life Oriented DSP Lab Modules
}

\section{Mr. Isaiah I. Ryan, Western Washington University}

Isaiah I. Ryan is currently a senior student in the Electronics Engineering Technology program at Western Washington University. His work focuses mainly on embedded system design, but he studies other topics as well such as digital signal processing. Isaiah enjoys applying theory from class and working together with his classmates to develop understanding of new material. Ultimately, he hopes to use his knowledge for the benefit of others.

\section{Aaron Cramer, Western Washington University}

Aaron Cramer holds the position of Instruction and Classroom Support Technician for Western Washington University's Electronics Engineering Technology/Electrical Engineering program. He earned his BS degree in Electronics Engineering Technology from Western in 2009. His prior industry experience includes Applications Engineer for Cypress Semiconductor and Production Technician for Bruker Elemental.

\section{Dr. Ying Lin, Western Washington University}

Ying Lin has been with the faculty of Engineering and Design Department at Western Washington University since September 2010 after she taught for two years at SUNY, New Platz. She received her MS in Applied Statistics and Ph.D. in Electrical Engineering from Syracuse University, NY, respectively. Her teaching interests include first-year Intro to Electrical Engineering, and upper-level communication systems and digital Signal Processing courses. Her research areas focus on statistical signal processing for wireless sensor network applications and secure communications in wireless networks. 


\title{
Real-time Real-life Oriented DSP Lab Modules
}

\begin{abstract}
:
In this paper, we present a sequence of engaging lab exercises that implement real-time real-life signal/data acquisition, analysis, and processing using MatLab, LabView, and NI myDAQ. Examples of these signals include real-time human voice and music signals. These lab modules are designed to enhance the existing lab components in the digital signal processing and/or digital communications curriculum in an Electronic Engineering Technology (EET) program at Western Washington University. The lab topics cover fundamentals of digital signal processing (DSP) such as real-time data acquisition, sampling, time-domain and frequency-domain analysis, and digital filtering. Noise analysis and removal examples have been also introduced in the lab modules. Moreover, advanced DSP techniques such as speech recognition have been incorporated in the labs to implement a voice-controlled DSP application as well.

Besides serving the EET curriculum, the labs developed in this work can be used as effective outreach tools. For instance, we have adopted these labs as demos to groups of $5^{\text {th }}$ graders of an annual Compass to Campus program in our institution to promote engineering and technology to young minds. These newly developed engaging lab demos will help to further attract and spark young students' interests in engineering and technology.
\end{abstract}

\section{Introduction}

Digital signal processing (DSP), an important field in Electrical engineering, embraces a broad spectrum of applications, ranging from speech encoding, synthesis, and recognition, image processing, digital/wireless communication systems, radar and sonar systems, control systems, to name a few. Most modern electronic gadgets use some DSP techniques. For instance, the "SIRI" function in iphone 4 uses DSP-based speech recognition algorithms. High quality headphones employs DSP-based noise cancelation techniques as well.

DSP has become an integral part of Electronic Engineering Technology (EET) and Electrical Engineering curricula at higher institutions worldwide. To fulfill a successful DSP curriculum, it is critical to complement lectures with well-designed hands-on laboratory exercises. It has been widely acknowledged that hands-on experiences improve teaching and learning efficiency and reinforce students' comprehension of abstract topics ${ }^{1,2,3,4}$. 


\section{Motivations and Objectives:}

Our existing MatLab-based DSP lab exercises, similar to many other DSP curriculum in other institutions ${ }^{5}$, use non-real-time signals that are either pre-recorded or generated in MatLab. Recently, there has been an increasing need of introducing real-time data acquisition and measurements and processing into the curriculum. According to a recent senior student survey, interest in this area of study has greatly increased. Members of the EET program Industry Advisory Board from local industry have suggested this area to be considered in our curriculum as well.

To meet such need, in this work, our goal is to develop a series of fun and engaging hands-on DSP laboratory exercises that embrace real-time real-life signal processing. The signals adopted in the newly developed labs are real-time and real-life signals, such as human voice or music signals input from a microphone. These types of signals have been often employed in existing DSP lab curriculum $^{2}$.

We strive to relate the DSP theories to real-life examples and make better connections between theory and practice. Specifically, these laboratory exercises facilitate students to achieve the following outcomes, but not limited to:

- Enhancing students' understanding of DSP fundamentals;

- Connecting theories learned in class to practice;

- Accumulating hands-on skills in practicing and implementing commonly used DSP algorithms and techniques;

- Getting familiar with popular software and hardware tools adopted in the DSP field.

The key topics covered in these labs include DSP fundamentals such as period sampling, timedomain analysis, frequency domain (spectrum) analysis, digital filtering, and noise analysis and removal. Moreover, some advanced DSP techniques such as speech recognition are also incorporated into the lab exercises. Details of these topics will be presented in later sections.

\section{Tools Adopted:}

A number of software and hardware tools have been selected in developing these hands-on labs. The software tools include MatLab and LabView.

MatLab, a powerful computing and simulation tool, has been widely used in DSP labs and projects $^{5,6}$. It serves well as a simulation tool for DSP algorithms. In this project, we have used MatLab for multiple tasks:

- To design filters and simulate the filter response.

- To realize math script within a LabView VI program.

On the other hand, LabView, a popular platform used in industry for real-time measurement and testing applications, is a suitable tool to accomplish real-time signal acquisition, analysis, and 
processing tasks. Due to these features, we have adopted LabView as the major software platform in developing these lab exercises. Other benefits of using LabView include that students get exposed to such a useful tool and have opportunities to build up their experience and skills which prepare them for their future career in this area.

In addition, the major hardware components used in these lab modules are data acquisition units such as NI myDAQ and a microphone.

\section{Summary of Developed Laboratory Modules}

In this work, we have developed two main LabView-based lab modules and each consists of a number of tasks or a sequence of lab exercises. We have also used a NI myDAQ for real-time data acquisition and digital output control.

The key topics covered in these lab modules are summarized in Table 1.

Table 1: Summary of Lab Module Topics

\begin{tabular}{|l|l|}
\hline \multicolumn{1}{|c|}{ Lab Module } & Topics (Tools used) \\
\hline \multirow{5}{*}{ Lab Module \#1 } & $\begin{array}{l}\text { Real-time input signal generation (LabView/NI DAQ/function } \\
\text { generator) }\end{array}$ \\
\cline { 2 - 3 } & Signal time-domain analysis (LabView) \\
\cline { 2 - 3 } & Signal frequency-domain analysis (LabView) \\
\cline { 2 - 2 } & Noise analysis and removal through digital filtering (LabView) \\
\cline { 2 - 2 } & Digital filter design and simulation (MatLab) \\
\cline { 2 - 2 } & Sound recording and replay (LabView) \\
\cline { 2 - 2 } & $\begin{array}{l}\text { Various sound effect such as echo } \\
\text { (MatLab and LabView) }\end{array}$ \\
\hline & $\begin{array}{l}\text { Speech recognition } \\
\text { (Microsoft windows API, LabView, and a microphone) }\end{array}$ \\
\cline { 2 - 2 } & $\begin{array}{l}\text { Voiced-controlled fan system } \\
\text { (LabView, NI DAQ, a microphone, a MOSFET, and a mini-fan) }\end{array}$ \\
\hline
\end{tabular}

In the following sections, we present detailed lab objectives, tasks, and lab results.

\section{Lab Module \#1}

This module focuses on fundamental topics in DSP.

Lab objectives and pedagogical goals: 
This lab module aims to provide students hands-on opportunities to:

○ Practice time-domain and frequency-domain analysis of a real-time signal;

- Conduct frequency spectrum analysis;

- Acquaint with noise analysis;

- Design and apply digital filters to remove noise component;

- Implement common audio processing techniques such as generating echo effects.

It consists of several lab exercises, as presented below.

\section{1: Time-domain analysis and frequency-domain analysis of a single tone signal}

\section{$\underline{\text { Lab Tasks: }}$}

- Input a real-time sine signal into LabView. This input can be either a sine signal generated from a physical function generator that connects to LabView through a DAQ or a sine signal created by the corresponding LabView palette.

- Provide the time-domain plot and frequency spectrum plot, respectively. Analyze the timedomain and the frequency spectrum.

\section{$\underline{\text { Lab results: }}$}

Figure 1 shows the snapshots of the two plots for a sine signal with frequency of $1 \mathrm{kHz}$. As clearly demonstrated from the spectrum graph, the signal indeed is a $1 \mathrm{kHz}$ sine signal given that it has one peak at $1000 \mathrm{~Hz}$. 


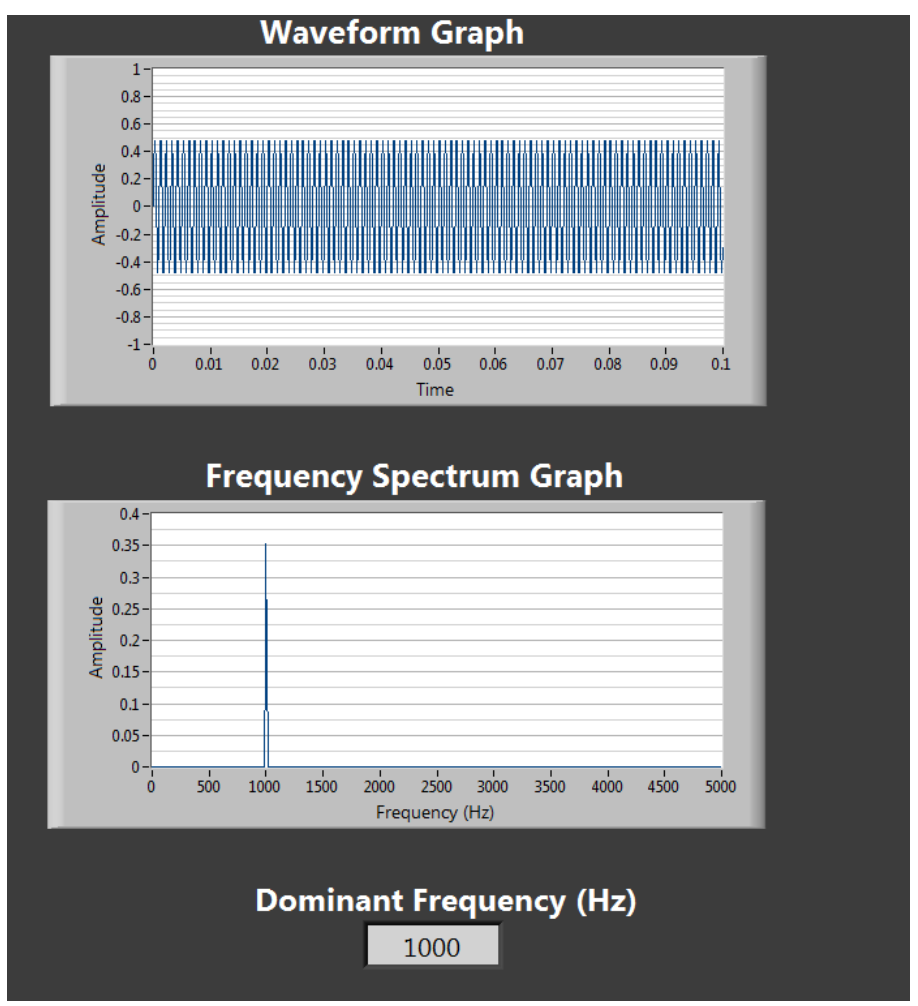

Figure 1: Snapshot of the time-domain plot and frequency spectrum plot for A1. The corresponding LabView GUI snapshot is provided in Figure 2. 


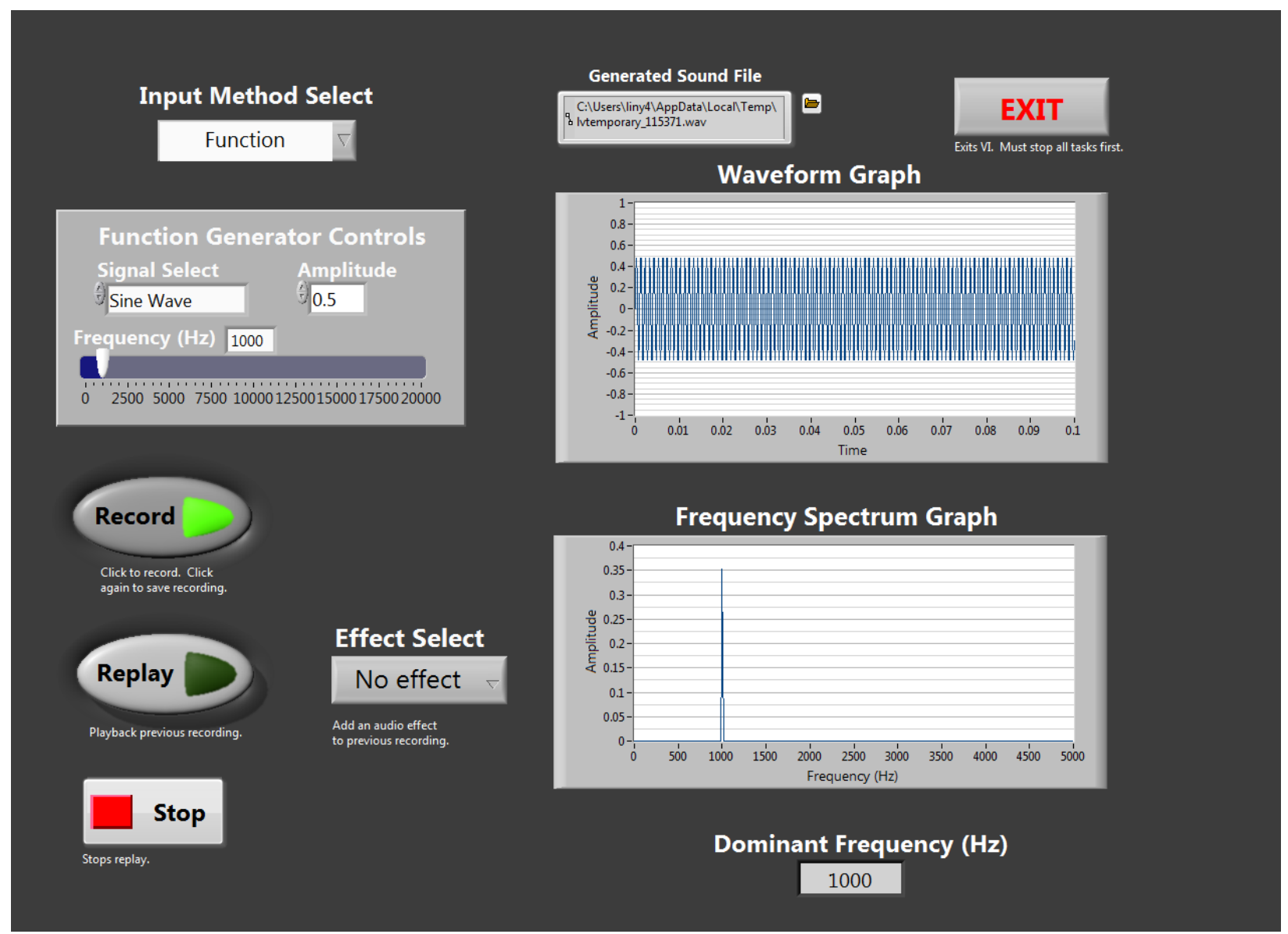

Figure 2: Snapshot of the LabView GUI block diagram for A1 with the selected input method as "function generator from a LabView palette".

\section{2: Time-domain analysis and frequency-domain analysis of human voice/music}

\section{Lab Tasks:}

- The input can be either human voice or a piece of music taken from a microphone that connects to the PC running LabView.

- Provide the time-domain plot and frequency spectrum plot, respectively. Analyze what major frequency components are included in the input signal.

\section{$\underline{\text { Lab results: }}$}

Figure 3 shows the snapshots of the two plots for a clip of human voice. As predicted, it is difficult to tell the frequency information from the time-domain plot, however, from the frequency spectrum plot, it is clear that human voice typically consists of lower frequency components. This is consistent with theory.

Specifically, for this particular user, the major components are below or around $500 \mathrm{~Hz}$. 


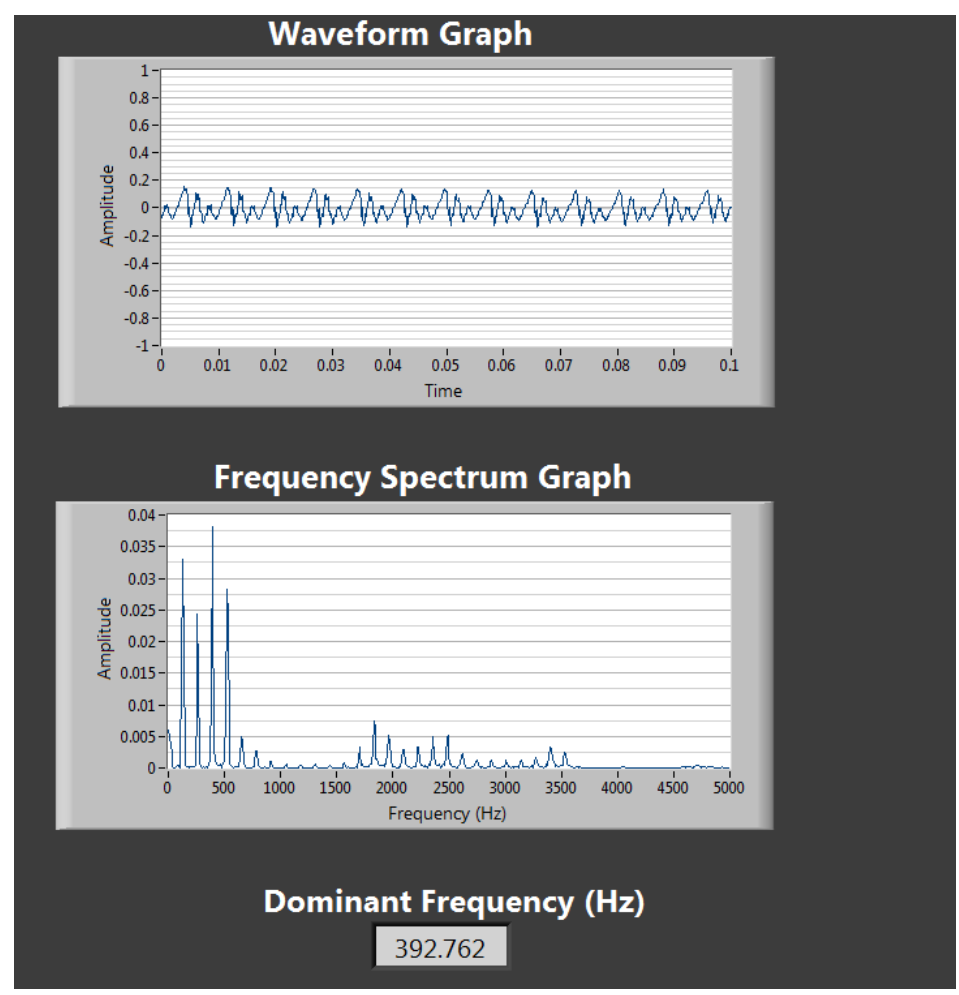

Figure 3: Snapshot of the time-domain plot and frequency-spectrum plot of human voice.

In Figure 4, the LabView GUI block diagram shows that the selected input method as "Microphone". 


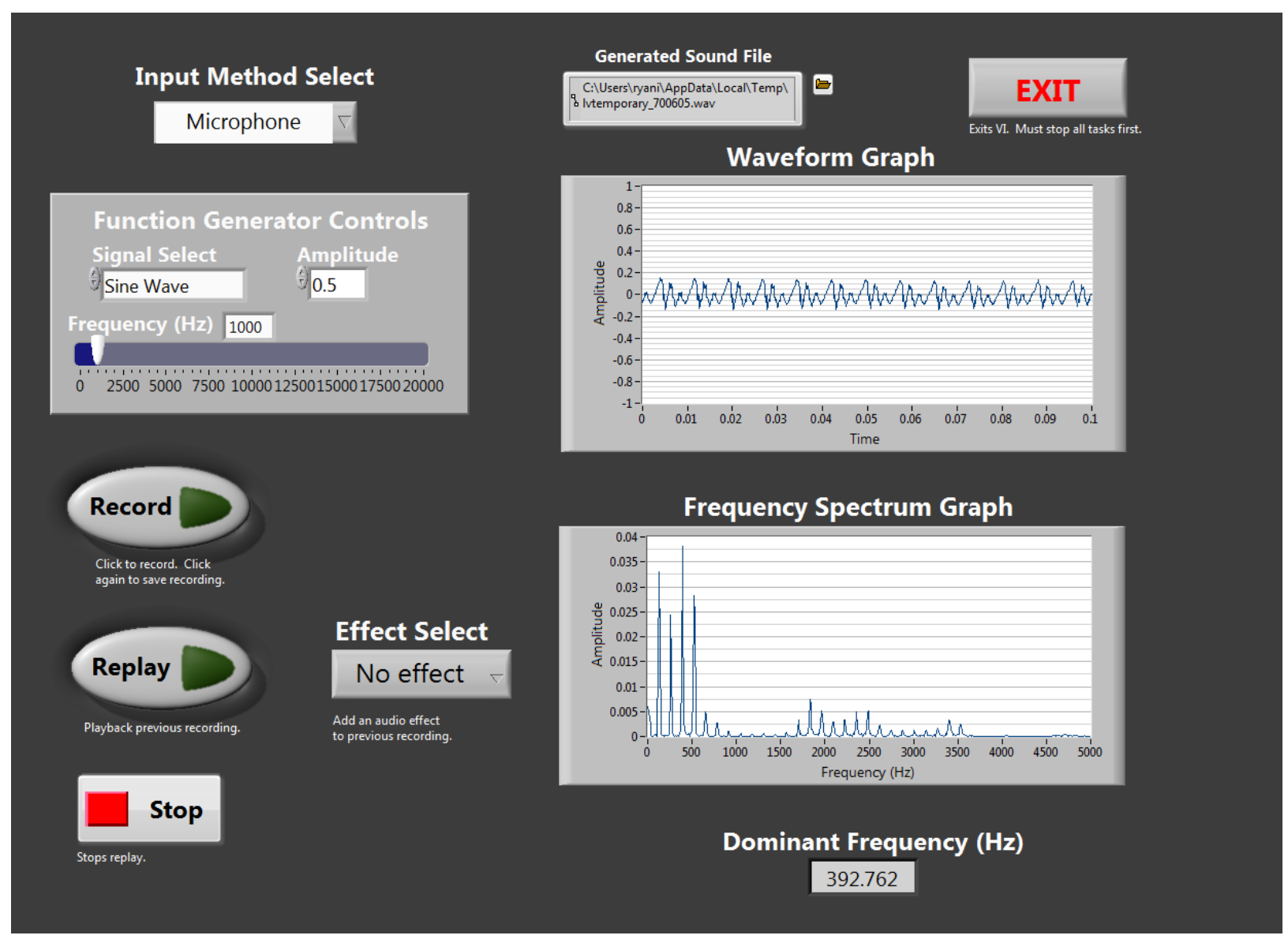

Figure 4: Snapshot of the LabView GUI block diagram for A1 with the selected input method as "Microphone".

\section{3: Noise analysis and removal through digital filtering}

$\underline{\text { Lab Tasks: }}$

- Create the desired (wanted) signal. It can be from any of aforementioned input sources such as either human voice or a piece of music taken from a microphone or a sine tone from a function generator.

- Create the noise signal which is a single tone signal. It can be generated from a physical function generator or from a LabView palette.

- Mix the desired signal with the noise signal.

- Plot the time-domain plot and frequency-spectrum plot, respectively.

$\bigcirc$ Analyze the frequency components of the mixed signal and design a digital filter to remove the noise.

- Display the filter frequency response.

$\circ$ Apply the filter and provide the recovered signal time-domain plot and frequency spectrum plot. 
$\circ$ Analyze the quality of the recovered signal after filtering.

\section{$\underline{\text { Lab results: }}$}

Figure 5 provides the snapshots of the time-domain and frequency spectrum plot for the mixture signal of human voice and the noise signal of a $1 \mathrm{kHz}$.

As predicted, from the time-domain analysis, it is difficult to tell the frequency information and differentiate between the desired signal and the noise signal in the time-domain plot, however, useful information may be obtained through conducting the frequency-domain analysis.

In this particular example, it appears that the $1 \mathrm{kHz}$ noise is dominant with a large peak on the spectrum plot. Again the human voice occupies mostly the lower frequency range (blow 500 $\mathrm{Hz}$ ). Such difference provides a good mechanism for possible noise removal.

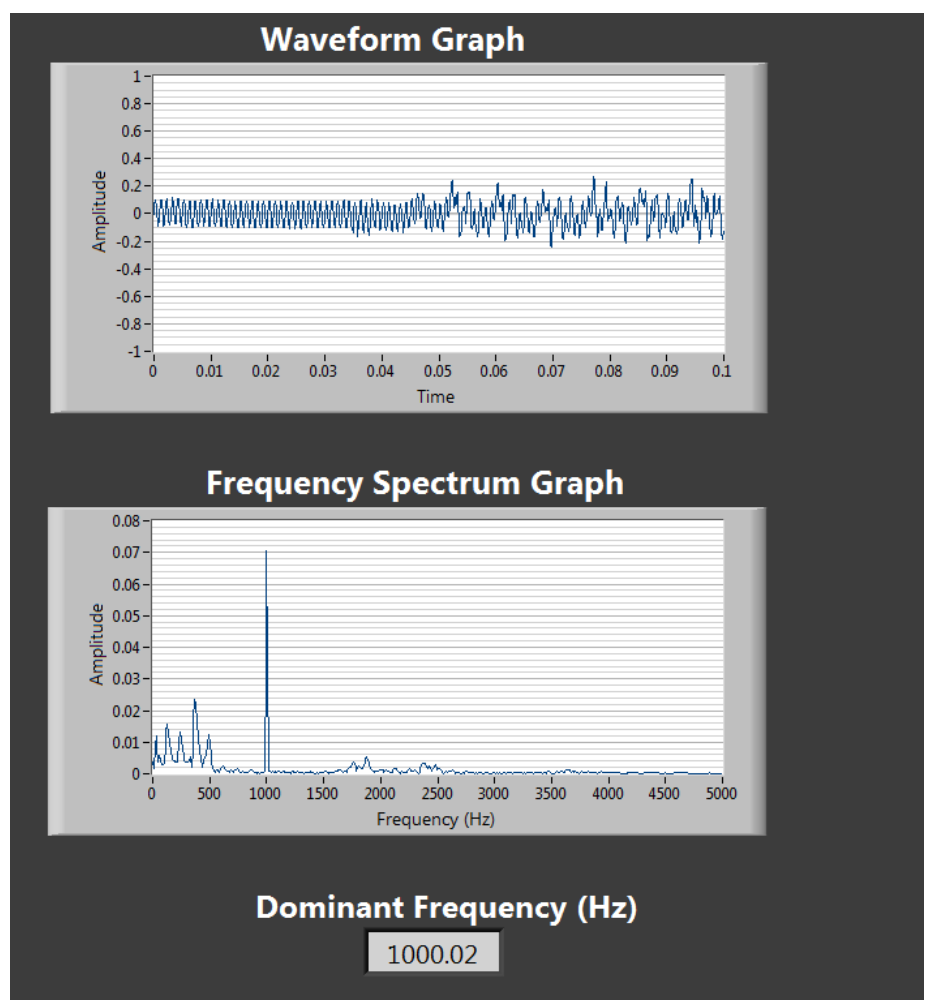

Figure 5: Time-domain plot and frequency-spectrum of the mixture of human voice and a $1 \mathrm{kHz}$ single tone noise signal.

To remove the noise signal, a notch filter centered at $1 \mathrm{kHz}$ can be applied to the mixture signal.

In this project, we have used MatLab to design and simulate the digital filter. The corresponding frequency response is shown in Figure 6. 


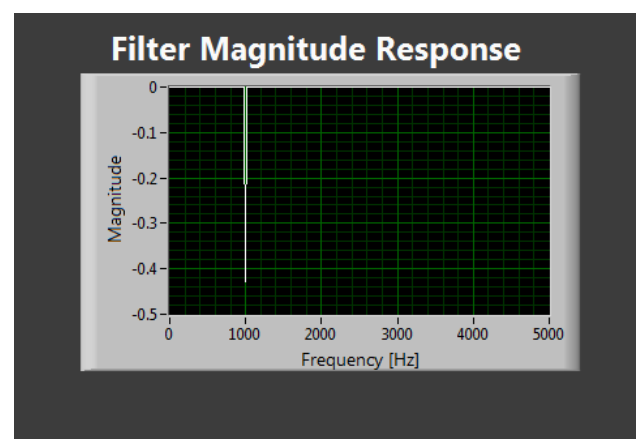

Figure 6: Frequency response of a notched filter with notched frequency of $1 \mathrm{kHz}$.

The recovered signal after the notched filter is applied is shown in Figure 7. Clearly, the $1 \mathrm{kHz}$ noise signal has been successfully removed as shown from the spectrum plot in Figure 7 . The sound quality is comparable to the original sound signal and is thus acceptable as well.

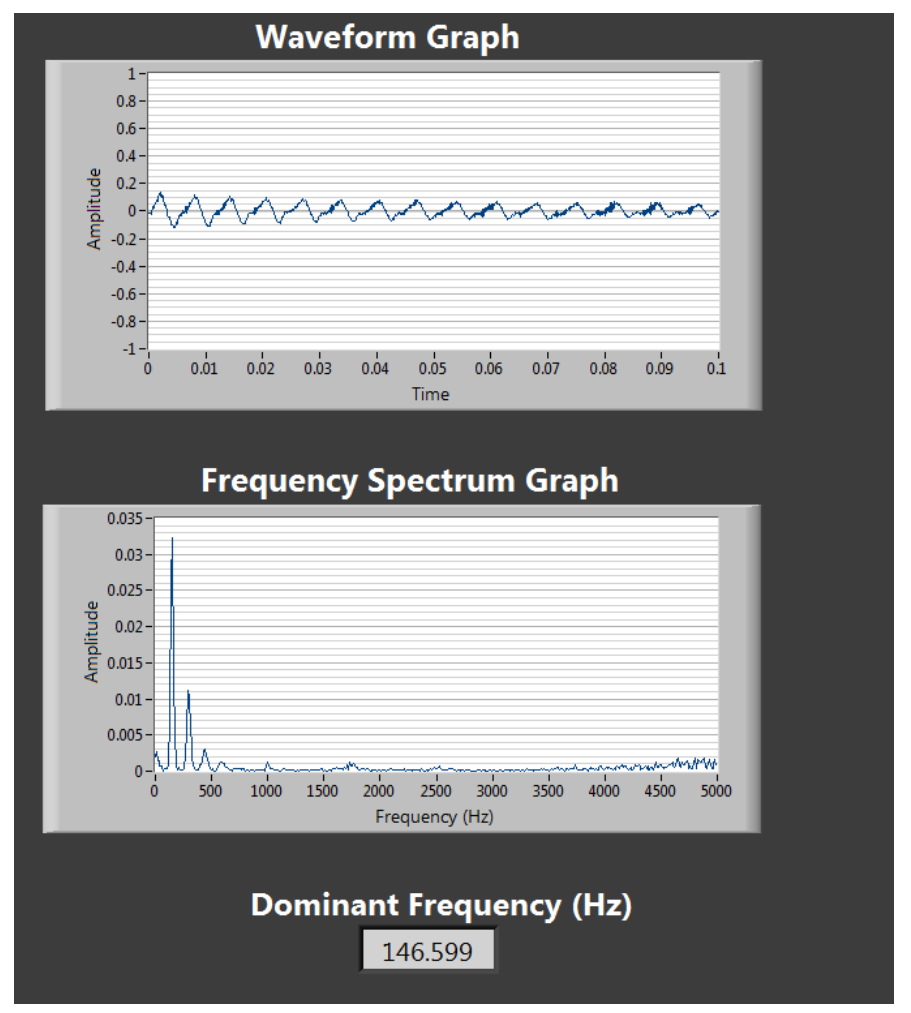

Figure 7: Snapshot of the time-domain plot and frequency spectrum of the recovered signal after filtering.

Figure 8 shows the snapshot of the complete LabView GUI block diagram for the noise and filter operations described above. 


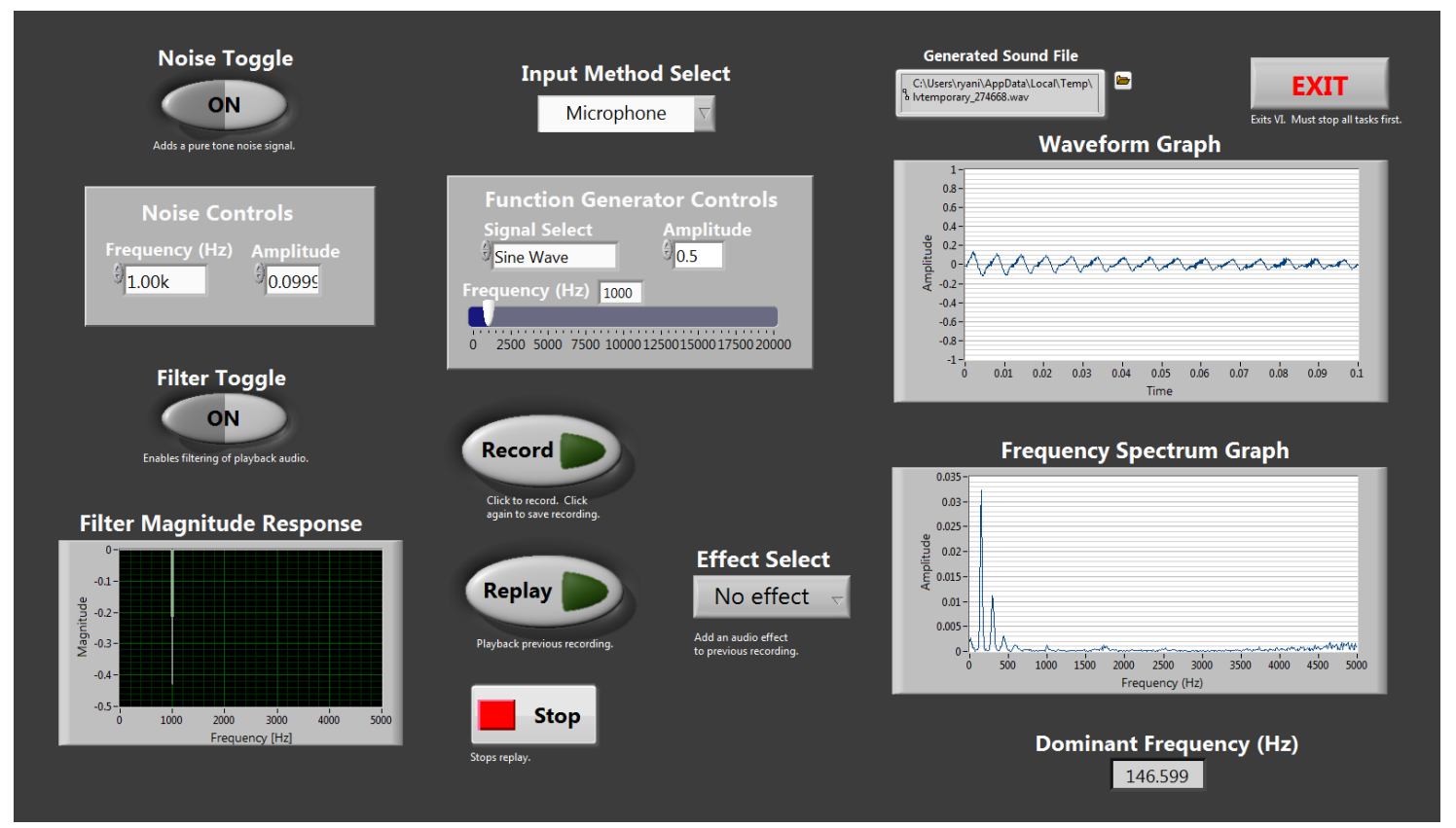

Figure 8: Snapshot of the LabView GUI block diagram for noise removal

\section{4: Audio signal processing in LabView}

\section{$\underline{\text { Lab Tasks: }}$}

- Create a sound recorder function. The input can be any of the input methods. Save the recorded sound to a file.

- Implement the sound replay function.

- Create an echo effect. The input signal can be from any of the three input methods.

- Create a higher pitch doubling effect.

\section{$\underline{\text { Lab results: }}$}

Figure 8 also shows the snapshots of these two functions realized in LabView GUI. The echo effect has been created according the block diagram depicted in Figure 9.

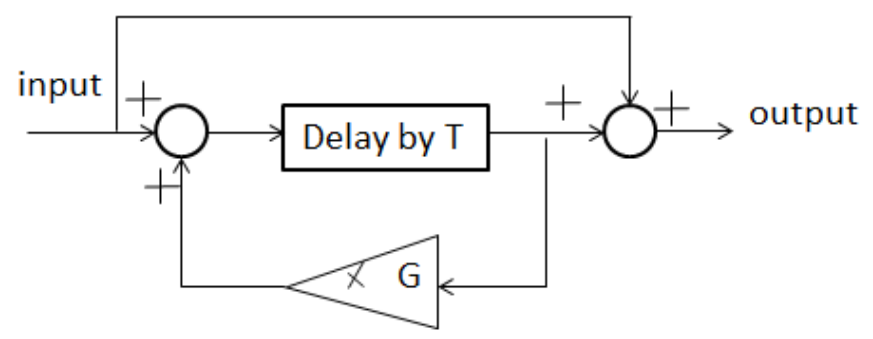

Figure 9: Block diagram of an echo system 
The complete LabView VI program structure for lab module \#1 is shown in Figure 10.

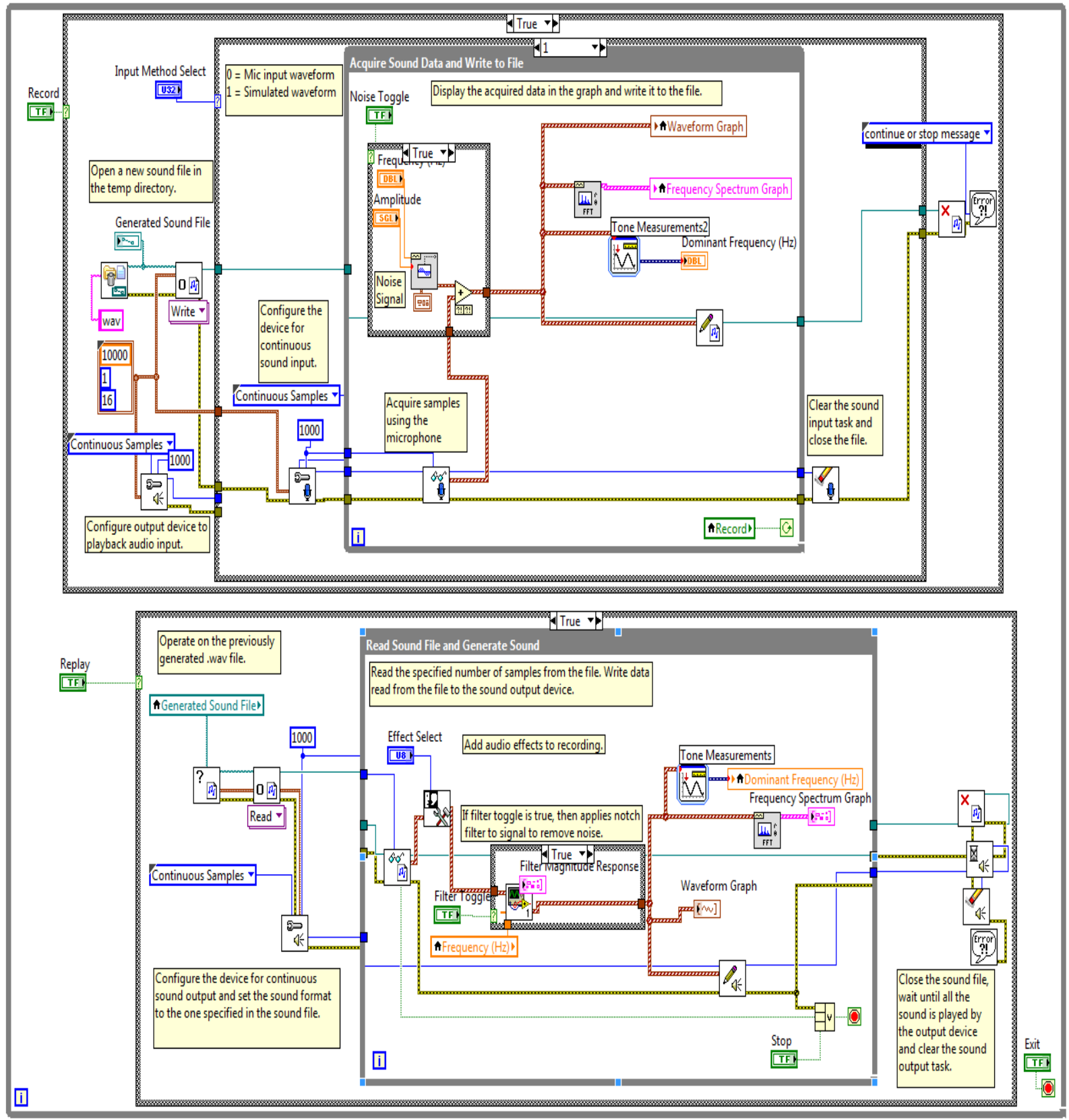

Figure 10: Complete LabView VI program structure for lab module \#1. 


\section{Lab Module \#2}

This module focuses on practical DSP applications. Specifically, it implements a voice-controlled fan system which uses speech recognition and LabView digital output control through a NI DAQ unit. Software tools include LabView and Microsoft windows functions. Major hardware components adopted are NI DAQ, a mini-fan, a microphone, a MOSFET, and a breadboard.

\section{Lab objectives and pedagogical goals:}

This lab module aims to provide students hands-on opportunities to:

○ Practicing common DSP techniques such as speech recognition in real-time applications;

○ Implementing a DSP-driven fan control system through voice commands;

- Getting familiar with using NI DAQ and LabView for real-time system control.

This lab module consists of several lab exercises, as described below.

\section{1: Speech recognition algorithm in LabView}

\section{Lab Tasks:}

- Implement a speech recognition algorithm in LabView. This algorithm is taken from a Microsoft windows API function and is linked into LabView.

○ Test the speech algorithm.

\section{Lab results:}

Figure 11 shows the LabView VI program structure for speech recognition. Note that this VI program is rather simple due to the fact that it calls a windows API function directly to implement the speech recognition functionality. 


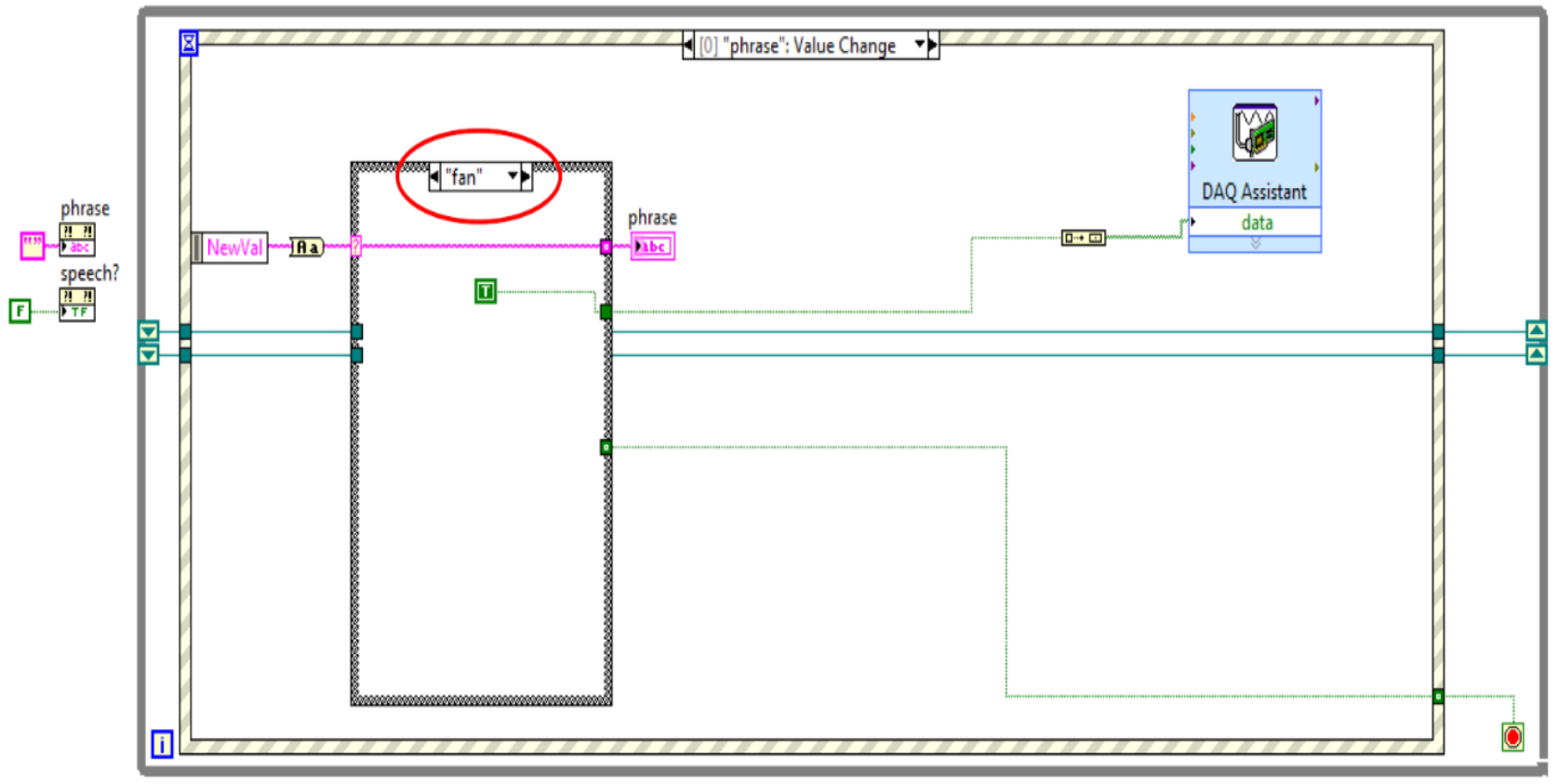

Figure 11: LabView VI program structure for voice recognition and fan control.

\section{2: Voice-controlled fan system}

\section{$\underline{\text { Lab Tasks: }}$}

- Use voice commands to drive a digital output to the NI DAQ to operate /stop a fan.

\section{Lab results:}

When a user speaks "fan", the fan will start to operate; it will stop working when a user speaks "stop". Testing results from students who took the "digital communications" course in spring 2014 have indicated that the correct recognition rate is high and the system works as designed.

The actual LabView VI program structure for the voice-controlled fan system is depicted in Figure 11. Figure 12 demonstrates the physical system component connections.

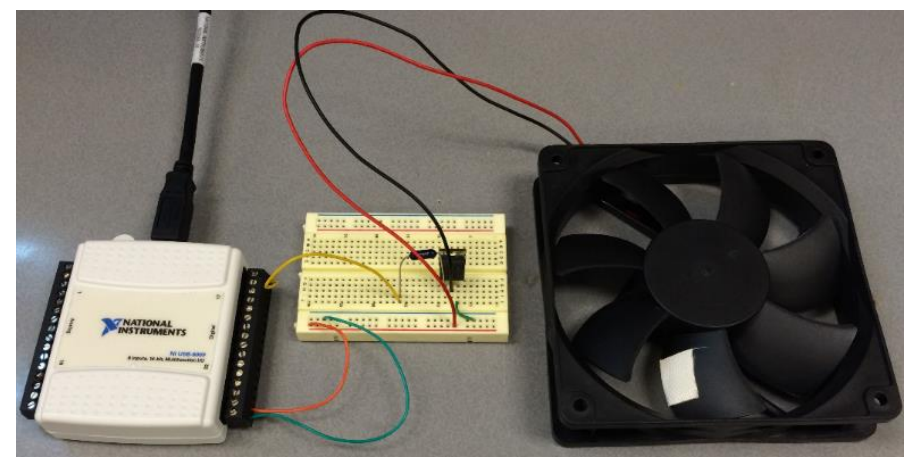

Figure 12: A photo of component connections for the voice-controlled fan system. 


\section{Assessment Results}

This section provides assessment results to demonstrate the effectiveness of these lab modules. The assessment data include lab results, student survey, and feedback from the 2014 "compass to campus" outreach program participants in our institution.

As presented in preceding sections (Section III and Section IV), the corresponding lab results are consistent with the theory and effectively demonstrate the DSP principles in various topics.

Student survey collected from students who took the DSP class in fall 2014 is summarized in Table 2. Majority of students acknowledged that the lab topics were engaging and facilitated their understanding of the concepts.

Table 2: Student Survey Questions on DSP Lab Modules (Total \# of responses = 14)

\begin{tabular}{|l|l|l|l|l|}
\hline \multicolumn{1}{|c|}{ Questions } & $\begin{array}{l}\text { Strongly } \\
\text { Agree }\end{array}$ & Agree & Disagree & $\begin{array}{l}\text { Strongly } \\
\text { disagree }\end{array}$ \\
\hline $\begin{array}{l}\text { The hands-on labs enhance my understanding of } \\
\text { the concepts learned in class such as DFT, frequency- } \\
\text { domain spectrum analysis, and FIR filters. }\end{array}$ & $57 \%$ & $43 \%$ & $0 \%$ & $0 \%$ \\
\hline $\begin{array}{l}\text { The hands-on labs such as recovering desired signal } \\
\text { from the noisy mixed signals are beneficial and help to } \\
\text { get acquaints with the filter concept. }\end{array}$ & $42 \%$ & $58 \%$ & $0 \%$ & $0 \%$ \\
\hline $\begin{array}{l}\text { Overall speaking, the hands-on labs effectively } \\
\text { complement the lecture materials. }\end{array}$ & $35 \%$ & $58 \%$ & $7 \%$ & $0 \%$ \\
\hline $\begin{array}{l}\text { The hands-on labs are engaging and provide a good } \\
\text { educational experience. }\end{array}$ & $42 \%$ & $51 \%$ & $7 \%$ & $0 \%$ \\
\hline
\end{tabular}

In addition, feedback from participants ( $5^{\text {th }}$ graders) of an outreach event ("compass to campus" in October, 2014) was extremely positive and encouraging. The $5^{\text {th }}$ grade visitors were all excited about trying out the audio processing demos (Lab module \#1) such as the echo effect and a highfrequency doubling effect demos. They asked questions about how to produce these effects and requested to add other fun audio effects as well. This experience attests that the developed lab modules can be effective tools in stimulating and attracting young students to this field.

\section{Conclusion}

In this work, we have successfully developed a number of hands-on laboratory exercises for digital signal processing curriculum using LabView, Matlab, Windows API function, and data acquisition 
units. These labs offer students opportunities to practice DSP fundamentals with real-time real-life DSP applications in speech/audio/music. The resulting labs are practically fun and educationally engaging.

In addition to the academic impact, the labs developed in this work will also benefit an outreach effort to inspire interests from younger students (e.g., $5^{\text {th }}$ graders who participate in the annual compass to campus program in our institution) in STEM education. These labs will also serve as demos to showcase Engineering and Technology to prospective students, visitors, and guests.

Moreover, the lab modules, the software, and hardware employed to complete this project can be adapted to future laboratory and project developments in other areas such as Digital Communications, and Digital Control Systems curricula.

\section{References:}

1. Feisel, L. D., and Rosa, A. J., "The Role of the Laboratory in Undergraduate Engineering Education." Journal of Engineering Education 94(1): 121-130, 2005.

2. Buket D. Barkana, “Curriculum Development of an Audio Processing Laboratory Course." Signal \& Image Processing, March, 2011.

3. S. Shelke, M. Date, S. Patkar, R. Velmurugan, P. Rao, "A Remote Lab for Real-time Digital Signal Processing." Education and Research Conference (EDERC), 5th European DSP, January, 2012.

4. Radojka Krneta, Marjan Milosevic, Djordje Damnjanovic, Danijela Milosevic, "Matching Learning Styles to Different Type of DSP Laboratory Experiments.” IEEE Global Engineering Education Conference (EDUCON), April, 2014.

5. Sanjit Mitra, "Digital Signal Processing, a Computer-Based Approach”, McGraw-Hill Science/Engineering/Math, 3rd edition, 2005, ISBN: 0072865466.

6. James McClellan, and et al, "DSP First: A Multimedia Approach”, Prentice Hall, 1st edition, 1998, ISBN:

0132431718 . 